Supporting Information for:

\title{
Sub-nanometric roughness affects deposition and mobile adhesion of Escherichia coli on silanized glass surfaces
}

\author{
Sumedha Sharma, ${ }^{1}$ Yuly Andrea Jaimes-Lizcano, ${ }^{1}$ Ryan B. McLay, ${ }^{1}$ Patrick C. Cirino, ${ }^{1}$ and \\ Jacinta C. Conrad ${ }^{1,2^{*}}$ \\ ${ }^{1}$ Department of Chemical and Biomolecular Engineering and ${ }^{2}$ Department of Petroleum \\ Engineering, University of Houston, Houston, TX 77204-4004
}

\section{Supporting movies online:}

Movie S1: Deposition of E. coli MC1061 on DTS-modified glass (22 $\mathrm{mPa}$ ) showing mobile adhesion of cells on the surface and immobile surface association. The time interval between frames is $3 \mathrm{~s}$ and the playback speed is 5 frames/s.

Movie S2: Deposition of E. coli MC1061 on PTS-modified glass (22 mPa) showing the absence of mobile adhesion. The time interval between frames is $3 \mathrm{~s}$ and the playback speed is 5 frames/s.

Movie S3: Deposition of E. coli MC1061 on FDTS-modified glass (22 $\mathrm{mPa}$ ) showing mobile adhesion of cells on the surface and immobile surface association. The time interval between frames is $3 \mathrm{~s}$ and the playback speed is 5 frames/s.

Movie S4: Deposition of E. coli MG1655 $\mathrm{fimA}$ on DTS-modified glass (22 $\mathrm{mPa}$ ) showing mobile adhesion of cells on the surface and immobile surface association. The time interval between frames is $3 \mathrm{~s}$ and the playback speed is 5 frames/s. 


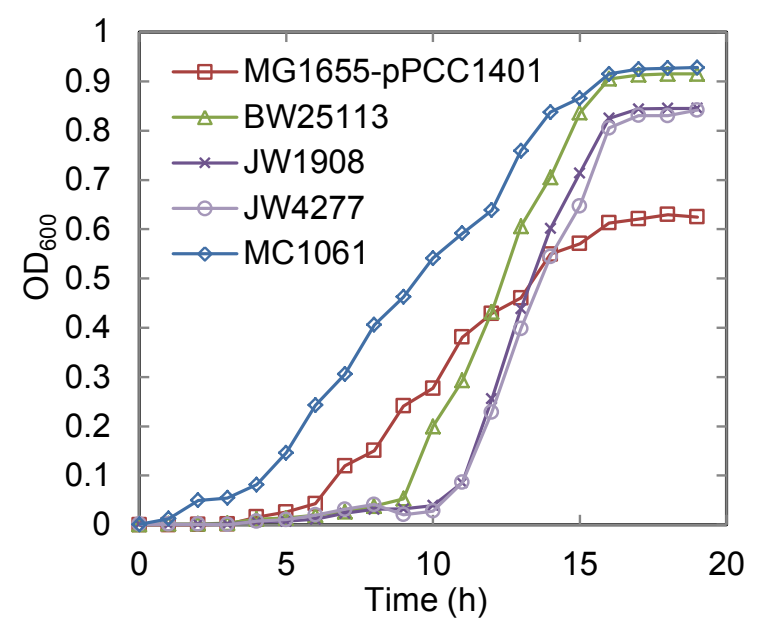

Figure S1. Growth curves of E. coli bacteria strains used in this study.

\section{S1. Flagella and fimbriae expression assays}

The expression of fimbriae in all bacterial strains used in this study was tested using a fluorescence antibody conjugation assay and confocal microscopy. Bacterial suspensions were prepared following the same protocols used in the deposition experiments and reported in the manuscript. $50 \mu \mathrm{L}$ of the sample suspension was mixed with pAB49 rabbit anti-fimA antibody, ${ }^{1}$ which attached to any type 1 fimbriae that were present on the cells. After $10-15$ minutes, $10 \mu \mathrm{L}$ of $0.2 \mathrm{mg} / \mathrm{mL}$ AlexaFluor568 conjugated, goat-derived anti-rabbit antibody was added and allowed to react in the dark for 10 minutes. Labeled fimbriae were visualized using confocal microscopy (VTEye, Visitech) equipped with a laser excitation source of wavelength $561 \mathrm{~nm}$. Images were acquired at $100 \times$ magnification. We observed fimbriae expression only in strain MG1655-pPCC1401 (Figure S2), which was engineered to express fimbriae when induced with IPTG. No fimbriae expression was seen on any of the other bacteria strains under the culture conditions used in the deposition experiments. 


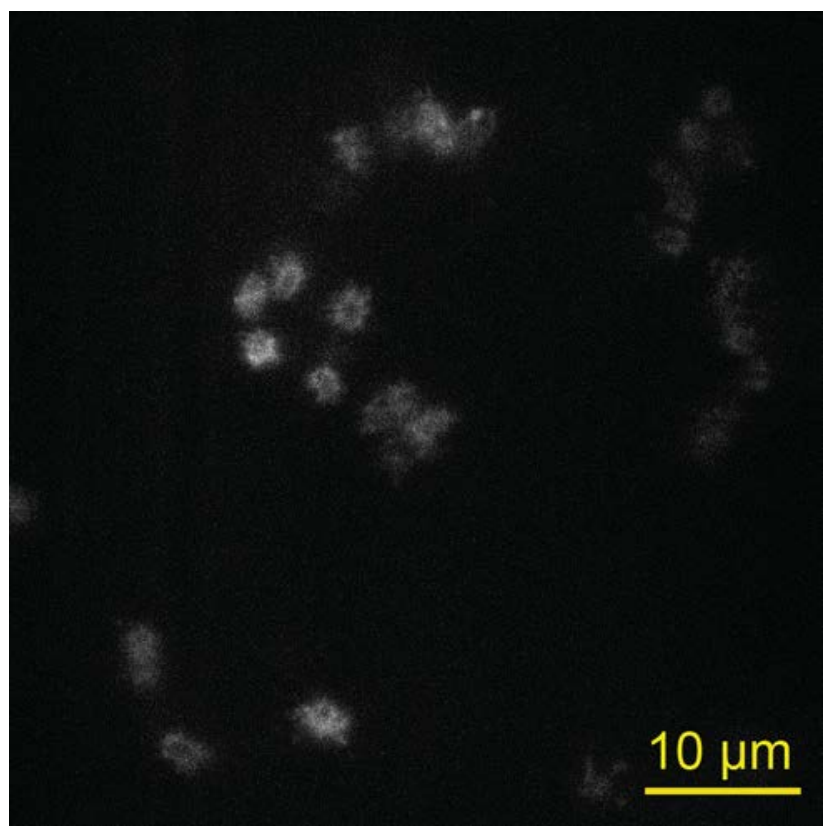

Figure S2. Confocal micrograph of MG1655-pPCC1401 showing bristle brush type expression of fimbriae on the cell surface

\section{S2. Transmission electron microscopy (TEM) imaging of bacteria}

TEM imaging of all bacterial strains was used to visualize expression of two bacterial appendages, flagella and fimbriae. For all strains except MG1655-pPCC1401, cell suspensions for imaging were prepared following the same protocol used for flow experiments. For imaging, $2-5 \mu \mathrm{L}$ of bacterial suspension was adsorbed onto a freshly glow-discharged carbon-coated grid $\left(\right.$ PELCO $\left.{ }^{\circledR}\right)$. The sample grid was washed gently with water and stained with $2 \%(\mathrm{w} / \mathrm{v})$ uranyl acetate. Imaging was done using a JEOL 1200 EX TEM operated at an acceleration voltage of $100 \mathrm{kV}$. Electron micrographs were recorded at calibrated magnifications using a 3k slow-scan CCD camera (model 15C, SIA) and are shown in Figure S3. Flagella expression was observed in all strains except JW1908, which is a flagella-deficient mutant of BW25113. Fimbriae were observed only for MG1655-pPCC1401 strain grown in static conditions with IPTG induction. All other strains did not express fimbriae. 


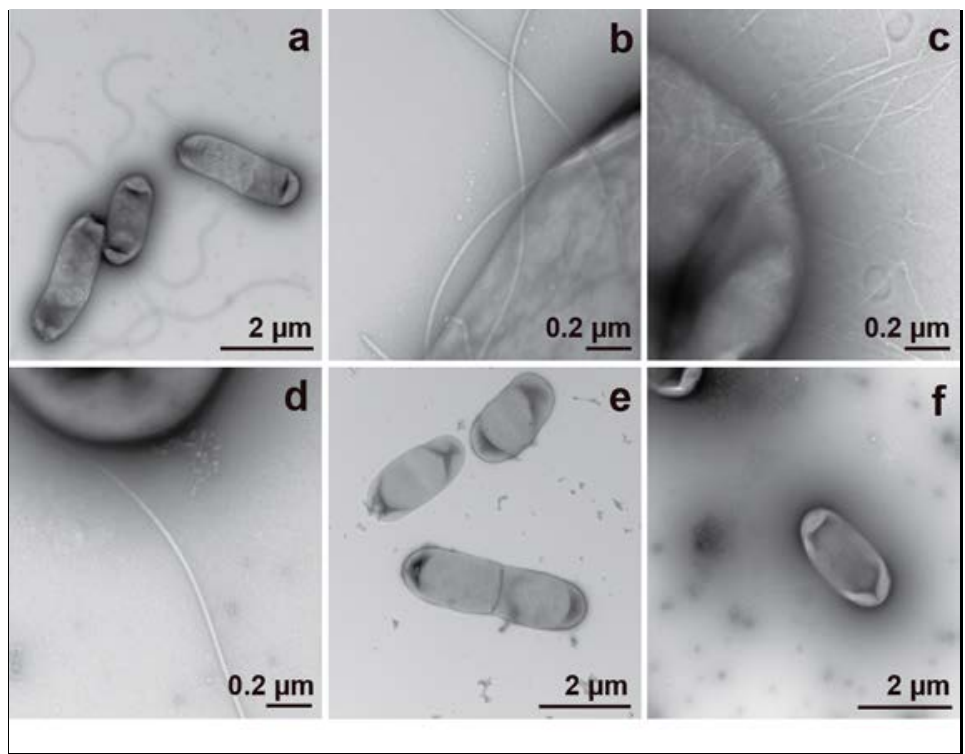

Figure S3. TEM micrographs for bacterial strains (a) MC1061, showing flagella and absence of fimbriae expression; (b) MG1655 $\triangle$ fimA, showing presence of flagella but not fimbriae; (c) MG1655-pPCC1401, showing fimbriae expression; (d) BW25113, showing flagella and absence of fimbriae expression; (e) JW1908, showing absence of both flagella and fimbriae; (f) JW4277, showing presence of flagella.

\section{S3. XPS characterization of silanized substrates}

The presence of chemical groups expected for self-assembled layers of silanes on glass was confirmed using X-ray photoelectron spectroscopy (Physical Electronics Model 5700) using Al Ka X-ray source $(1486.6 \mathrm{eV})$ under ultrahigh vacuum. The takeoff angle was $45^{\circ}$ and the analyzer spot diameter was $800 \mu \mathrm{m}$. Survey and high-resolution spectra were recorded at pass energies of 187.85 and $23.5 \mathrm{eV}$, respectively. The binding energy scales were referenced to $284.6 \mathrm{eV}$, corresponding to the maximum intensity for a C 1s spectrum. Spectral analysis and peak fitting was performed using the Multipak software package (Multipak V5.0A, Physical Electronics, Inc.). Atomic compositions were estimated from the high-resolution scans. Fluorination of the glass slides treated with FDTS and FPTS was confirmed by the presence of F1s peak at around $688.7 \mathrm{eV}$, as shown in the survey spectra (Figure $\mathrm{S} 4$ ). 


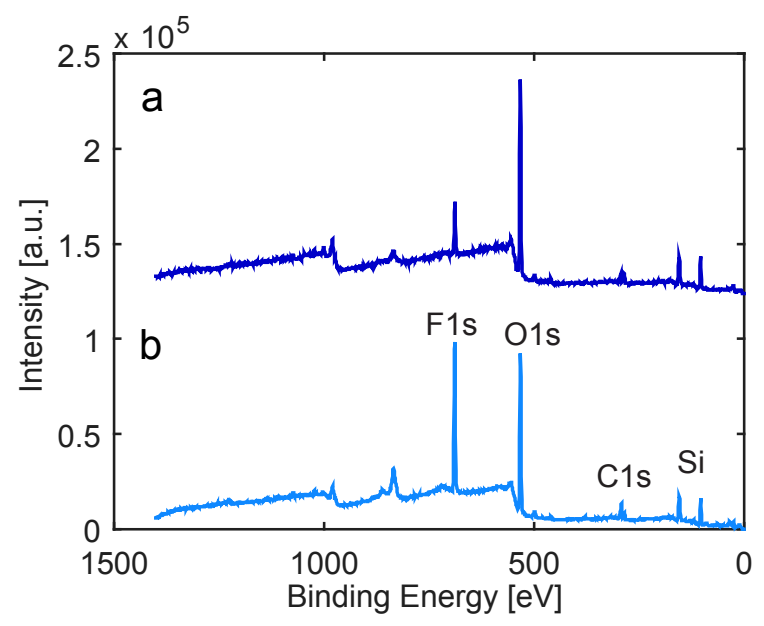

Figure S4. XPS survey spectra of (a) FPTS and (b) FDTS surfaces. The distinct F1s peak at $688.7 \mathrm{eV}$ confirms the attachment of the fluorinated silanes.

The chemical compositions of the fluorinated substrates were calculated from the high-resolution spectra and reported in Table S1. No peaks related to the chlorine species appeared in any spectrum, confirming that the trichlorosilane groups of the silanes reacted completely with the hydroxyl groups on the glass substrate. ${ }^{2}$ The measured F/C mass ratio is 1.28 and 0.49 for FDTS and FPTS, respectively. These ratios are different from the ideal values for a single monolayer; we attributed this difference to presence of additional $\mathrm{C}$ expected from exposure of surfaces to air.

Table S1. Percentage atomic composition determined by XPS high-resolution spectra for the fluorinated surfaces.

\begin{tabular}{cccccc}
\hline Substrate & C1s & O1s & F1s & Si2p & K2p \\
\hline FPTS & 17.83 & 48.18 & 8.82 & 23.24 & 1.92 \\
FDTS & 18.78 & 35.70 & 24.10 & 18.14 & 3.27 \\
\hline
\end{tabular}

The survey spectra for the alkylsilane surfaces are presented in Figure S5. Again, no peaks related to the chlorine species appeared in any spectrum, confirming that the trichlorosilane groups of the silanes reacted completely with the hydroxyl groups on the glass 
substrate. High-resolution spectra were acquired for $\mathrm{C} 1 \mathrm{~s}, \mathrm{O} 1 \mathrm{~s}$, and $\mathrm{Si} 2 \mathrm{p}$, and were used to calculate the chemical composition of the surfaces (Table S2).

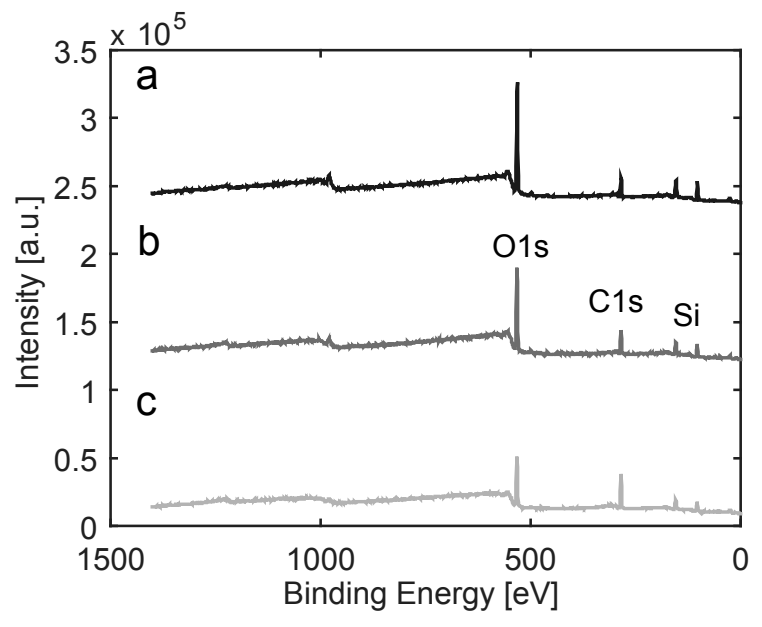

Figure S5. XPS survey spectra of the methyl-terminated silanized surfaces: (a) PTS (b) DTS and (c) OTS. The $\mathrm{C} 1 \mathrm{~s}$ peaks at $284.6 \mathrm{eV}$ reflect contributions of attached alkylchlorosilanes.

Table S2. Atomic composition determined by XPS high-resolution spectra for the methylterminated surfaces.

\begin{tabular}{cccc}
\hline Substrate & C1s & O1s & Si2p \\
\hline PTS & 19.01 & 55.52 & 25.47 \\
DTS & 30.96 & 43.21 & 25.83 \\
OTS & 55.89 & 29.01 & 15.10 \\
\hline
\end{tabular}

\section{S4. Extended DLVO calculation}

Extended DLVO theory was used to estimate the interaction between the bacteria and silanized substrates as detailed in our earlier work. ${ }^{3}$ Briefly, the interaction of a rigid and spherical bacterium $(b)$ with a planar surface $(s)$ in a medium $(m)$ was calculated as ${ }^{4}$

$$
\Delta G^{\text {total }}=\Delta G^{L W}+\Delta G^{A B}+\Delta G^{E L}
$$

where the superscripts indicate the contributions from Lifshitz-van der Waals $(L W)$, acid base $(A B)$, and electrostatic $(E L)$ interactions. 
The surface potential for bacteria and surfaces was estimated from measurements of zeta potential. To approximate the zeta potential of the flat silanized surfaces, we measured the zeta potential of silanized silica nanoparticles. The zeta potentials of the bacteria suspensions and of silanized nanoparticles suspended in $154 \mathrm{mM} \mathrm{NaCl}$ was measured using a NanoBrook ZetaPALs (Brookhaven Instruments) $\zeta$-potential analyzer and reported in Tables 2 and 3 in the manuscript.

To assess the cell surface characteristics of bacteria, we measured the contact angle of water and two other test solvents on bacterial lawns. The bacterial lawns were prepared by spreading $100 \mu \mathrm{L}$ of concentrated bacterial suspension onto a clean glass slide kept during $24 \mathrm{~h}$ in a desiccator box. ${ }^{5}$ Static water contact angles of the cell lawns were measured using a Dataphysics OCA 15EC goniometer. Reported data correspond to at least eight spot measurements made on three different lawns. The water contact angles obtained for all the studied strains were similar, indicating comparable levels of cell surface hydrophobicity. Contact angles for two other test liquids, ethylene glycol (99\%, Alfa Aesar), and diiodomethane (99\%, Alfa Aesar) were also measured on these lawns (Table S3). The surface energies of the bacteria and of surfaces were calculated from the water contact angles using the method described by van Oss. $^{6}$

Table S3. Contact angles of E. coli strains used for surface tension calculations. The standard deviation was calculated from contact angle measurements on at least eight spots on three different bacterial lawns for each bacterial strain.

\begin{tabular}{cccc}
\hline Strain & $\begin{array}{c}\text { Water } \\
\text { contact angle } \\
{\left[{ }^{\circ} \text { (Std. Dev.) }\right.}\end{array}$ & $\begin{array}{c}\text { Diiodomethane } \\
\text { contact angle } \\
{\left[{ }^{\circ} \text { ] (Std. Dev.) }\right.}\end{array}$ & $\begin{array}{c}\text { Ethylene glycol } \\
\text { contact angle } \\
{\left[{ }^{\circ} \text { ] (Std. Dev.) }\right.}\end{array}$ \\
\hline MC1061 & $37(3)$ & $64(4)$ & $56(4)$ \\
BW25113 & $40(4)$ & $63(6)$ & $58(2)$ \\
JW4277 & $38(4)$ & $48(4)$ & $56(5)$ \\
JW1908 & $37(4)$ & $60(5)$ & $59(4)$ \\
\hline
\end{tabular}


Table S4. Values of surface energies used in xDLVO calculations.

\begin{tabular}{ccccc}
\hline Sample & $\boldsymbol{\gamma}_{\boldsymbol{i}}^{\boldsymbol{L W}}\left[\mathbf{m} \mathbf{J} / \mathbf{m}^{2}\right]$ & $\boldsymbol{\gamma}_{\boldsymbol{i}}^{+}\left[\mathbf{m J} / \mathbf{m}^{2}\right]$ & $\boldsymbol{\gamma}_{\boldsymbol{i}}^{-}\left[\mathbf{m J} / \mathbf{m}^{2}\right]$ & $\boldsymbol{\gamma}\left[\mathbf{m J} / \mathbf{m}^{2}\right]$ \\
\hline MC1061 & 27 & 0.11 & 72 & 32 \\
BW25113 & 27 & 0.16 & 70 & 34 \\
JW1908 & 35 & 0.80 & 68 & 50 \\
JW4277 & 28 & 0.41 & 75 & 40 \\
PTS & 27 & 0.18 & 6.2 & 29 \\
DTS & 22 & 0.010 & 1.4 & 23 \\
OTS & 20 & 0 & 0.3 & 20 \\
FPTS & 23 & 1.5 & 8 & 30 \\
FDTS & 11 & 0.22 & 2 & 12 \\
\hline
\end{tabular}

Table S5. The maximum attractive force $\left(F_{\max }^{\mathrm{xDLVO}}\right)$ estimated using the XDLVO theory for different combinations of surfaces and bacterial strains.

\begin{tabular}{ccccc}
\hline \multicolumn{5}{c}{$\boldsymbol{F}_{\max }^{\mathrm{xDLV0}}(\mathbf{N})$} \\
\hline & MC1061 & BW25113 & JW1908 & JW4277 \\
FPTS & $1.9 \times 10^{-8}$ & $1.5 \times 10^{-8}$ & $3.2 \times 10^{-8}$ & $2.3 \times 10^{-8}$ \\
FDTS & $-6.1 \times 10^{-14}$ & $-6.7 \times 10^{-14}$ & $-8.2 \times 10^{-14}$ & $-1.6 \times 10^{-13}$ \\
PTS & $3.6 \times 10^{-8}$ & $3.0 \times 10^{-8}$ & $5.0 \times 10^{-8}$ & $4.0 \times 10^{-8}$ \\
DTS & $5.6 \times 10^{-14}$ & $6.4 \times 10^{-14}$ & $6.2 \times 10^{-9}$ & $1.9 \times 10^{-13}$ \\
OTS & $-9.5 \times 10^{-15}$ & $-1.1 \times 10^{-14}$ & $-1.3 \times 10^{-14}$ & $-2.5 \times 10^{-14}$ \\
\hline
\end{tabular}

\section{S5. Atomic Force Microscopy}

Atomic force microscopy (AFM; Nanoscope IV, Digital Instruments) was used to measure the surface roughness of silanized glass surfaces. In a typical experiment, a $4 \mu^{2}$ area was scanned at $2 \mathrm{~Hz}$ in tapping mode in air using an n-type silicon cantilever ( $\mu$ Masch, resonance frequency $=320 \mathrm{kHz}$, force constant $=40 \mathrm{~N} / \mathrm{m}$ ). AFM images were analyzed using NanoScope $6.13 \mathrm{R} 1$ software to calculate roughness parameters, including average surface roughness $\left(\mathrm{R}_{\mathrm{a}}\right)$, root-mean-square roughness $\left(\mathrm{R}_{\mathrm{q}}\right)$, ten-point average roughness $\left(\mathrm{R}_{\mathrm{z}}\right.$, defined as 
the difference in height between the average of the five highest peaks and the five lowest valleys along a profile), and mean peak-to-valley profile roughness $\left(\mathrm{R}_{\max }\right)$.

Table S6. Roughness measurements of silanized glass surfaces after preparation.

\begin{tabular}{ccccc}
\hline \multicolumn{5}{c}{ Roughness (nm) } \\
\hline Sample & $\mathrm{R}_{\mathrm{a}}$ & $\mathrm{R}_{\mathrm{q}}$ & $\mathrm{R}_{\mathrm{z}}$ & $\mathrm{R}_{\max }$ \\
FPTS & $0.56(0.07)$ & $0.7(0.1)$ & $1.4(0.3)$ & $7(2)$ \\
FDTS & $0.12(0.01)$ & $0.16(0.02)$ & $0.20(0.02)$ & $1.2(0.1)$ \\
PTS & $0.52(0.06)$ & $0.68(0.08)$ & $1.3(0.1)$ & $6.4(0.9)$ \\
DTS & $0.14(0.03)$ & $0.17(0.03)$ & $0.31(0.04)$ & $2.3(0.4)$ \\
OTS & $0.4(0.1)$ & $0.51(0.2)$ & $0.7(0.1)$ & $9(2)$ \\
\hline
\end{tabular}

To evaluate if changes to the surface roughness occurred over the duration of the experiment, silanized surfaces were immersed into $0.9 \% \mathrm{NaCl}$ for up to $30 \mathrm{~min}$; this time corresponds to the maximum duration of a bacterial deposition experiment.

Table S7. Roughness measurements of silanized glass surfaces after immersion in $0.9 \% \mathrm{NaCl}$.

\begin{tabular}{cccc}
\hline & \multicolumn{3}{c}{$\mathbf{R}_{\mathbf{q}}$ Roughness $(\mathbf{n m})$} \\
\hline Sample & After silanization & $\begin{array}{c}\text { After immersion in } \\
0.9 \% \mathrm{NaCl} \text { for } 15 \mathrm{~min}\end{array}$ & $\begin{array}{c}\text { After immersion in } \\
0.9 \% \mathrm{NaCl} \text { for } 30 \mathrm{~min}\end{array}$ \\
FPTS & $0.73(0.1)$ & $0.56(0.05)$ & $0.60(0.03)$ \\
FDTS & $0.16(0.02)$ & $0.12(0.01)$ & $0.16(0.02)$ \\
PTS & $0.68(0.08)$ & $0.53(0.1)$ & $0.57(0.2)$ \\
DTS & $0.17(0.03)$ & $0.18(0.02)$ & $0.20(0.04)$ \\
OTS & $0.5(0.2)$ & $0.34(0.05)$ & $0.34(0.03)$ \\
\hline
\end{tabular}

The roughness of DTS increased $17 \%$ after immersion during $30 \mathrm{~min}$ in the saline solution. No change in roughness was observed for FDTS surfaces. FDTS and DTS surfaces were still significantly smoother than any of the other studied surfaces. 


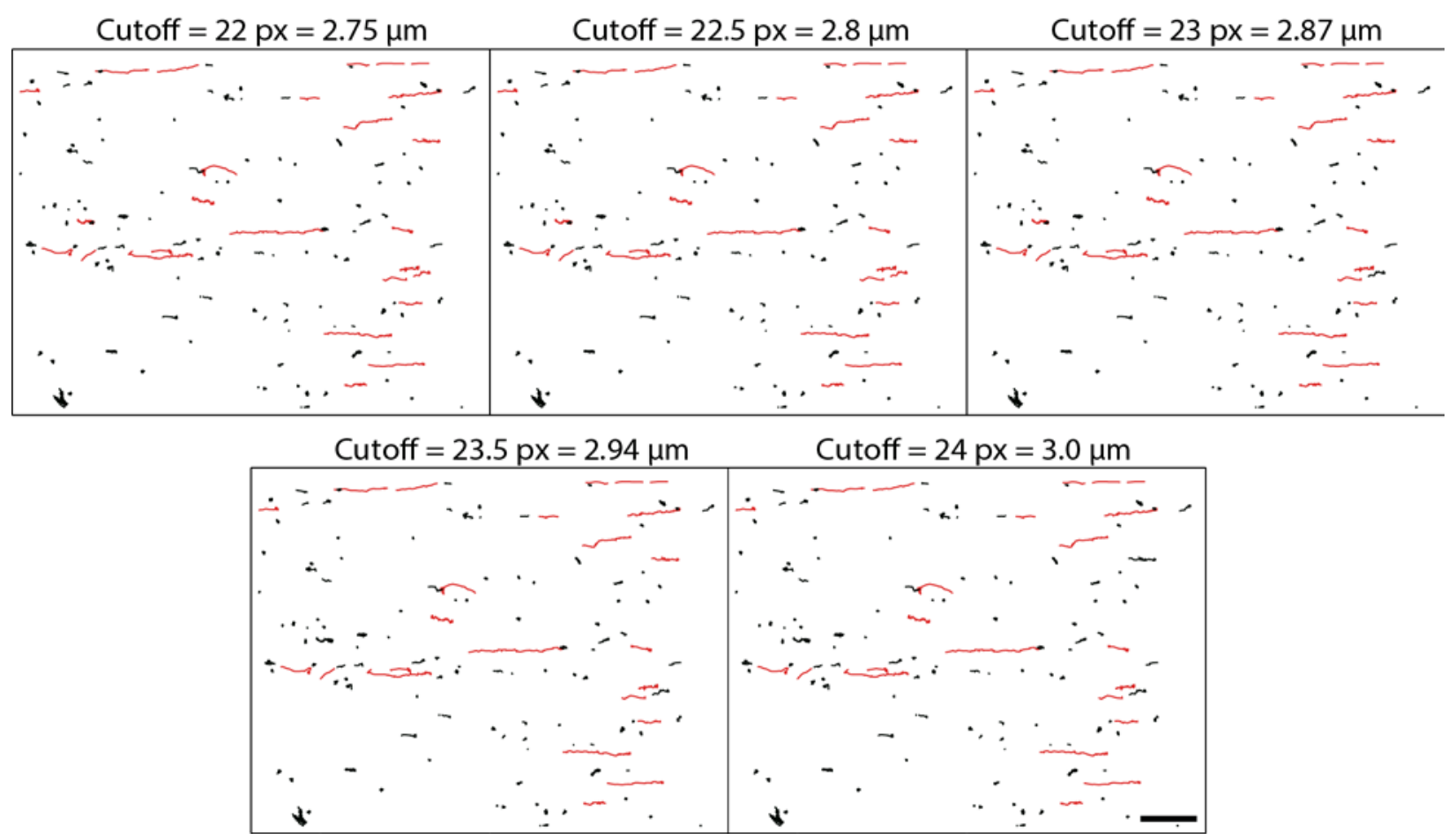

Figure S6. The trajectories for mobile (shown in red) and non-mobile cells (shown in black) on a DTS-modified glass substrate (shear stress $22 \mathrm{mPa}$ ) for different values of displacement cut-off are compared. The cut-off is defined as the minimum end-to-end displacement of a bacterium used to classify a cell as undergoing mobile adhesion. In the manuscript, we used a cutoff of 23 pixels (approximately 1.5 times the average bacterial length) to define mobile cells. Changing the cut-off values by 1 pixel more or less than the chosen definition does not change the percentage of cells exhibiting mobile adhesion behavior. Scale bar is $10 \mu \mathrm{m}$.

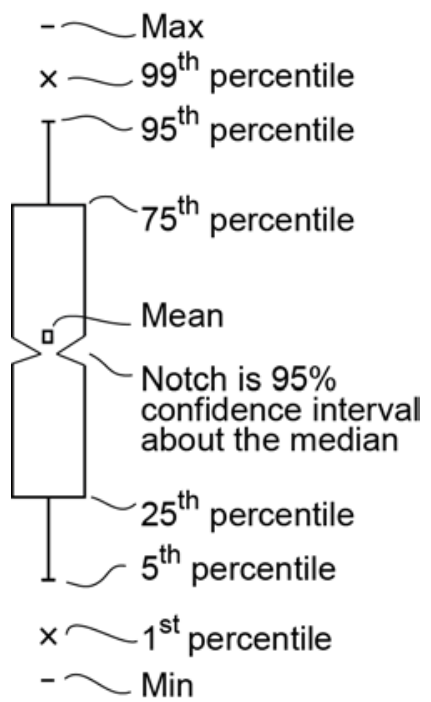


Figure S7. Legend defining the notched-box-plot used for reporting data in Figures $9-11$ in the paper.

\section{S6. Hydrodynamic force for particle touching a surface}

The hydrodynamic drag force is calculated from the equation given by O'Neil. ${ }^{7}$

Table S8. Hydrodynamic force calculations considering the bacteria as a sphere of equivalent volume.

\begin{tabular}{ccc}
\hline $\begin{array}{c}\mathrm{Q} \\
\left(\mathrm{m}^{3} / \mathrm{s}\right)\end{array}$ & $\begin{array}{c}V_{R} \\
(\mathrm{~m} / \mathrm{s})\end{array}$ & $\begin{array}{c}F_{h} \\
(\mathrm{~N})\end{array}$ \\
\hline $2.1 \times 10^{-9}$ & $2.2 \times 10^{-6}$ & $4.4 \times 10^{-14}$ \\
$1.7 \times 10^{-8}$ & $1.8 \times 10^{-5}$ & $3.5 \times 10^{-13}$ \\
$5.0 \times 10^{-8}$ & $5.3 \times 10^{-5}$ & $1.1 \times 10^{-12}$ \\
\hline
\end{tabular}

In this table, $V_{\mathrm{R}}$ is the fluid velocity at center of the particle and $\mathrm{F}_{h}$ is the hydrodynamic drag force.

\section{References}

1. Tchesnokova, V.; Aprikian, P.; Kisiela, D.; Gowey, S.; Korotkova, N.; Thomas, W.; Sokurenko, E. Type 1 Fimbrial Adhesin FimH Elicits an Immune Response That Enhances Cell Adhesion of Escherichia Coli. Infection and Immunity 2011, 79, 3895-3904.

2. Chen, J.-K.; Ko, F.-H.; Hsieh, K.-F.; Chou, C.-T.; Chang, F.-C. Effect of Fluoroalkyl Substituents on the Reactions of Alkylchlorosilanes with Mold Surfaces for Nanoimprint Lithography. J. Vac. Sci. Technol., B 2004, 22, 3233-3241.

3. Sharma, S.; Conrad, J. C. Attachment from Flow of Escherichia Coli Bacteria onto Silanized Glass Substrates. Langmuir 2014, 30, 11147-11155.

4. Van Oss, C. J.; Good, R.; Chaudhury, M. The Role of Van Der Waals Forces and Hydrogen Bonds in "Hydrophobic Interactions" between Biopolymers and Low Energy Surfaces. Journal Of Colloid and Interface Science 1986, 111, 378-390.

5. Harimawan, A.; Rajasekar, A.; Ting, Y.-P. Bacteria Attachment to Surfaces - Afm Force Spectroscopy and Physicochemical Analyses. J. Colloid Interface Sci. 2011, 364, 213-218.

6. van Oss, C. J. Acid-Base Interfacial Interactions in Aqueous Media. Colloids and Surfaces A: Physicochemical and Engineering Aspects 1993, 78, 1-49.

7. O'Neill, M. E. A Sphere in Contact with a Plane Wall in a Slow Linear Shear Flow. Chemical Engineering Science 1968, 23, 1293-1298. 